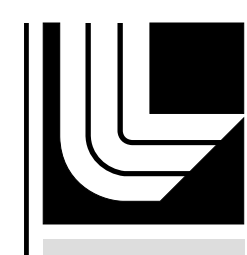

LAWRENCE LIVERMORE N A T IO N A L LABORATORY

\title{
Compound Nucleus Contributions to the Optical Potential
}

\section{LLNL-PROC-401271}

I. J. Thompson, F. S. Dietrich, J. E. Escher, M. Dupuis

February 12, 2008

Compound-Nuclear Reactions and Related Topics Fish Camp, CA, United States October 22, 2007 through October 26, 2007 
This document was prepared as an account of work sponsored by an agency of the United States government. Neither the United States government nor Lawrence Livermore National Security, LLC, nor any of their employees makes any warranty, expressed or implied, or assumes any legal liability or responsibility for the accuracy, completeness, or usefulness of any information, apparatus, product, or process disclosed, or represents that its use would not infringe privately owned rights. Reference herein to any specific commercial product, process, or service by trade name, trademark, manufacturer, or otherwise does not necessarily constitute or imply its endorsement, recommendation, or favoring by the United States government or Lawrence Livermore National Security, LLC. The views and opinions of authors expressed herein do not necessarily state or reflect those of the United States government or Lawrence Livermore National Security, LLC, and shall not be used for advertising or product endorsement purposes. 


\title{
Compound Nucleus Contributions to the Optical Potential
}

\author{
I.J. Thompson*, F.S. Dietrich*, J. E. Escher* and M. Dupuis ${ }^{\dagger}$ \\ ${ }^{*}$ Lawrence Livermore National Laboratory, P.O. Box 808, L-414, Livermore, CA 94551, USA \\ ${ }^{\dagger}$ T-16, Los Alamos National Laboratory, Los Alamos, NM 87545, USA
}

\begin{abstract}
An ab-initio calculation of the optical potential for neutron-nucleus scattering has been performed by explicitly coupling the elastic channel to all the particle-hole (p-h) excitation states in the target. These $\mathrm{p}-\mathrm{h}$ states may be regarded as doorway states through which the flux flows to more complicated configurations, and (in the end) to long-lived compound nucleus resonances. The random-phase approximation (RPA) provides the linear combinations of $\mathrm{p}$ - $\mathrm{h}$ states that include the residual interactions within the target, and we show preliminary results for elastic flux loss using both $\mathrm{p}$-h and RPA descriptions of target excitations.
\end{abstract}

Keywords: optical potential, compound nuclear reactions, direct reactions

PACS: 24.10.Ht, 24.10.Eq, 25.40.Dn

A key input to theoretical descriptions of many nuclear reaction theories is the optical potential. For neutroninduced reactions, which are typically based on statistical reaction theories such as Hauser-Feshbach, the optical potential defines the reaction cross section. Optical potentials are also critically important for theories of breakup reactions such as CDCC, as well as direct reactions such as transfers. The latter are a cornerstone of indirect experimental methods such as the surrogate reaction approach. At present, empirical potentials fit to data are used. For nuclei lying outside the range of the fits, such as those produced at rare-isotope facilities, in the r-process and in advanced reactor applications, this can lead to unquantifiable uncertainties.

The optical potential is specified by the requirement that when used within a one-channel model for elastic scattering, it produces the observed elastic scattering angular distribution. That is, it contains implicitly the effects of all channels such as collective excitations, transfer reactions, particle-hole excitations, as well as the production of compound-nucleus resonances. All of these processes directly remove flux from the elastic channel and therefore contribute to the negative imaginary part of the elastic optical potential. Compound nucleus resonances may perhaps decay back to the elastic channel, giving what is called the 'compound elastic contribution', but this return is delayed by the long lifetime of those resonances and is therefore excluded from the optical potential. Optical potentials have traditionally been found by fitting elastic scattering angular distributions by adjusting the parameters of standard forms of central and spin-orbit forces with real and imaginary components.

It is therefore a challenge to calculate optical potentials $a b$-initio, using the results of nuclear structure models such as those calculated in the SciDAC project 'Building a Universal Nuclear Energy Density Func- tional' (UNEDF) [1]. Such an ab initio approach should perform coupled-channels calculations including all the theoretical excited states deemed important, and then find the effective optical interaction in the elastic channel that describes the overall effect of coupling to all those states. The UNEDF results will thus play an essential role in replacing the phenomenological inputs with theoretically predicted ones. We present new calculations of the optical potential, looking first at neutron scattering from a ${ }^{90} \mathrm{Zr}$ target with a beam energy of $40 \mathrm{MeV}$.

\section{MODELS OF NUCLEAR EXCITATION}

The primary interface between the structure and reaction theories is the transition densities generated between the ground state and a set of low-lying excited states. In our calculations we use the level structure and the transition densities from Random Phase Approximation (RPA) calculations.

The state vectors used to describe the Hartree-Fock basis are defined as

$$
|n l s j m t\rangle=\frac{\phi_{n l j t}(r)}{r} i^{l}\left[Y_{l}(\hat{\mathbf{r}}) \times \chi_{\frac{1}{2}}\right]_{j m} \chi_{\frac{1}{2} t}^{i s o}
$$

where the radial wave functions $\phi_{n l j t}(r)$ are harmonicoscillator wave functions. We associate the creation and annihilation operators $a_{n l s j m t}^{\dagger}$ and $a_{n l s j m t}$ with these state vectors. It is convenient to define a modified annihilation operator,

$$
\tilde{a}_{n l s j m t}=(-1)^{j-m} a_{n l s j m t},
$$

which is a spherical tensor of rank $j$ and projection $-m$.

We now define particle $(p)$ and hole $(h)$ states corresponding to orbitals above or below the Fermi surface, schematically indicated by $p>F$ and $h<F$, respec- 

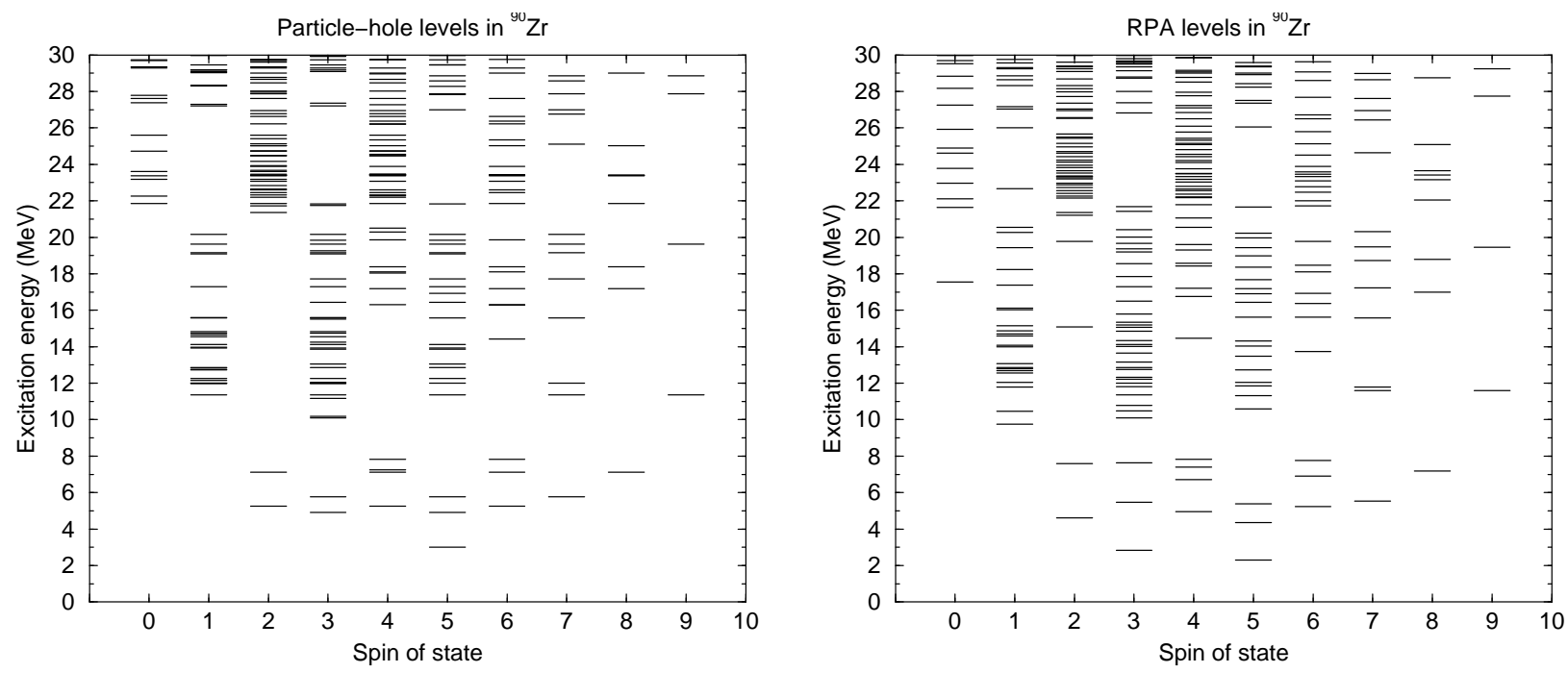

FIGURE 1. Single-particle (left) and RPA (right) levels calculated for ${ }^{90} \mathrm{Zr}$

tively. The symbol $p$ represents all quantum numbers except the magnetic projection; i.e. $p \equiv\left\{n_{p} l_{p} \frac{1}{2} j_{p} t_{p}\right\}$. The same definition applies to the hole states; replace $p$ by $h$.

With the above definitions, we define an operator that creates a particle-hole pair coupled to angular momentum $I$ and projection $M$,

$$
\mathscr{A}_{I M}^{\dagger}(p, \tilde{h})=\sum_{m_{p} m_{h}}\left(j_{p} m_{p} j_{h}-m_{h} \mid I M\right) a_{p m_{p}}^{\dagger} \tilde{a}_{h m_{h}} .
$$

From the Hermitian conjugate of this expression, we define another operator that destroys a particle-hole pair,

$$
\mathscr{A}_{I \bar{M}}(p, \tilde{h})=(-1)^{I-j_{p}+j_{h}} \sum_{m_{p} m_{h}}\left(j_{h} m_{h} j_{p}-m_{p} \mid I M\right) a_{h m_{h}}^{\dagger} \tilde{a}_{p m_{p}} .
$$

We define a boson operator $\Theta_{\alpha I M}^{\dagger}$ that creates the RPA state $|\alpha I M\rangle$ when applied to the correlated ground state $|0\rangle$, which is the vacuum for the RPA excitations. The $\alpha$ distinguishes the states of the same spin and parity. In terms of the particle-hole operators, the boson operator is

$$
\Theta_{\alpha I M}^{\dagger}=\sum_{p>F, h<F} X_{p h}^{\alpha I} \mathscr{A}_{I M}^{\dagger}(p, \tilde{h})-Y_{p h}^{\alpha I} \mathscr{A}_{I \bar{M}}(p, \tilde{h}),
$$

with coefficients $X_{p h}^{\alpha I}$ and $Y_{p h}^{\alpha I}$ that are found numerically by the RPA calculation for each excited state $\alpha I$.

For our first calculations we use the spherical HartreeFock initial state for ${ }^{90} \mathrm{Zr}$ calculated by Dupuis [2], which uses the Gogny D1S' force [3] with spin-orbit parameter $V_{s o}=-115 \mathrm{MeV}$. A harmonic oscillator basis up to $14 \hbar \omega$ was used, with $\hbar \omega=13.70 \mathrm{MeV}$ chosen to minimise the ${ }^{90} \mathrm{Zr}$ ground state energy. The RPA calculation of the spectrum removed the spurious $1^{-}$mode consisting of centre of mass motion, leaving the states shown on the right side of Fig. 1. Compared to the uncorrelated particle-hole spectrum on the left side of this figure, we do not yet see large or obvious changes except perhaps for the RPA $3^{-}$level becoming lower in energy.

\section{TRANSITION DENSITIES}

The transition density between states $\left|\alpha_{i} I_{i} M_{i}\right\rangle$ and $\left|\alpha_{f} I_{f} M_{f}\right\rangle$ is

$$
\begin{aligned}
& \rho_{S v}^{T q, \mathrm{fi}}\left(\mathbf{r}_{t}\right) \\
& =\left\langle\alpha_{f} I_{f} M_{f}\left|\sum_{n} \delta\left(\mathbf{r}_{t}-\mathbf{r}_{n}\right) \mathscr{S}_{S v}^{n} \mathscr{T}_{T q}^{n}\right| \alpha_{i} I_{i} M_{i}\right\rangle \\
& \equiv 4 \pi \sum_{L \mu J M}(L \mu S v \mid J M)\left(I_{i} M_{i} J M \mid I_{f} M_{f}\right) \rho_{L S J}^{T q}, \mathrm{fi}\left(r_{t}\right) Y_{L \mu}^{*}\left(\hat{\mathbf{r}}_{t}\right)
\end{aligned}
$$

where the $\mathscr{S}_{S v}^{n}$ and $\mathscr{T}_{T q}^{n}$ are the spin and isospin transition operators respectively. For $S=0$ or 1 ,

$$
\mathscr{S}_{00}=1 \quad \text { and } \quad \mathscr{S}_{1 v}=\sigma_{1 v},
$$

together with similar quantities $\mathscr{T}_{T q}$ for isospin,

$$
\mathscr{T}_{00}=1 \quad \text { and } \quad \mathscr{T}_{1 q}=\tau_{1 q},
$$

where $\sigma_{1 v}$ and $\tau_{1 q}$ are the spherical components of the vector of Pauli matrices. The coordinate-space radial density is

$$
\begin{array}{r}
\rho_{L S J}^{T q, \text { fi }}\left(r_{t}\right)=\frac{1}{\sqrt{2 I_{f}+1}}\left(\alpha_{f} I_{f} \| \sum_{n} \frac{1}{4 \pi} \frac{\delta\left(r_{t}-r_{n}\right)}{r_{t} r_{n}}\right. \\
\left.\left[Y_{L}\left(\hat{\mathbf{r}}_{n}\right) \times \mathscr{S}_{S}^{n}\right]_{J} \mathscr{T}_{T q}^{n} \| \alpha_{i} I_{i}\right),
\end{array}
$$



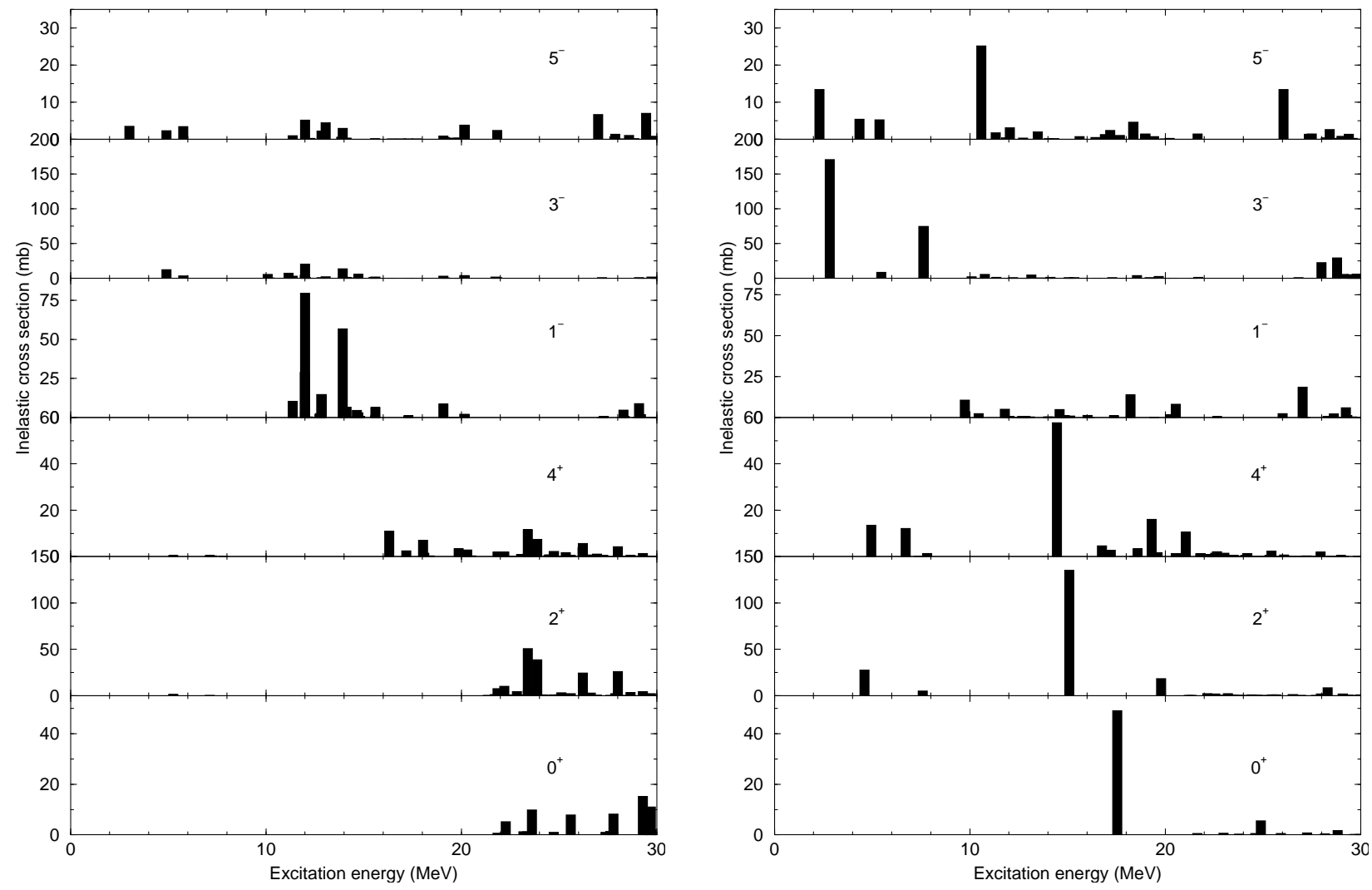

FIGURE 2. Single-particle (left) and RPA (right) inelastic cross sections for neutrons incident on ${ }^{90} \mathrm{Zr}$ at $40 \mathrm{MeV}$.

We can calculate the corresponding momentum-space density by the Fourier Bessel transform

$$
\begin{aligned}
\rho_{L S J}^{T q, \text { fi }}(q)= & \frac{1}{\sqrt{2 I_{f}+1}}\left(\alpha_{f} I_{f} \| \sum_{n} j_{L}\left(q r_{n}\right)\right. \\
& {\left.\left[Y_{L}\left(\hat{\mathbf{r}}_{n}\right) \times \mathscr{S}_{S}^{n}\right]_{J} \mathscr{T}_{T q}^{n} \| \alpha_{i} I_{i}\right) . }
\end{aligned}
$$

\section{EFFECTIVE FOLDING INTERACTION}

For an effective interaction between a scattering neutron and the nucleons in the nucleus, we use Love's effective $V_{n n}$ derived [4] from the M3Y interaction [5], with an approximate zero-range treatment of exchange effects. We reproduce the strength and range of $V_{n n}$ with a single Gaussian for our preliminary calculation. Using the Fourier transform $v^{S T}(q)$ of the effective interaction, the configuration space transition potential is

$$
U_{L S J}^{T q, \mathrm{fi}}(r)=\frac{2}{\pi} \int_{0}^{\infty} d q q^{2} j_{L}(q r) v^{S T}(q) \rho_{L S J}^{T q, \mathrm{fi}}(q) .
$$

We do not include any imaginary part in this effective interaction, as our aim is to include all the particle-hole excitations explicitly in our model.

\section{COUPLED CHANNELS CALCULATIONS}

When the coupling terms are local and not explicitly momentum dependent, we have to solve the coupled channels set of equations

$$
\begin{array}{r}
\left\{E-E_{c}^{\mathrm{ex}}+\frac{\hbar^{2}}{2 \mu_{c}} \frac{d^{2}}{d r^{2}}-\frac{l_{c}\left(l_{c}+1\right) \hbar^{2}}{2 \mu_{c} r^{2}}-V_{c c}^{\mathscr{J}}(r)\right\} u_{c}(r) \\
=\sum_{c^{\prime} \neq c} V_{c c^{\prime}}^{\mathscr{J}}(r) u_{c^{\prime}}(r),
\end{array}
$$

where $c$ identifies the target state $I_{c}$ with partial wave $l_{c}$ of orbital angular momentum for the neutron. The $E_{c}^{\mathrm{ex}}$ is the excitation energy of the RPA state, and $E$ is the centre of mass energy of the incident neutron.

The couplings $V_{c c^{\prime}}^{\mathscr{J}}$ for neutron scattering are given in terms of the $q=0$ parts of the mean over $T=0,1$ of the transition potential of Eq. (11) as

$$
\begin{aligned}
V_{c c^{\prime}}^{\mathscr{J}}(r)=i^{l^{\prime}}-l_{c} \sum_{L S J} & F_{L S J}^{\mathscr{J}}\left(l_{c} s_{c} j_{c} I_{c} ; l_{c^{\prime}} s_{c^{\prime}} j_{c^{\prime}} I_{c^{\prime}}\right) \hat{I}_{c}(-1)^{L+S-J} \\
& \times\left(\beta_{c} s_{c}\left\|\mathscr{S}_{S}\right\| \beta_{c^{\prime}} s_{c^{\prime}}\right) \sum_{T=0}^{1} \frac{1}{2} U_{L S J}^{T 0, c c^{\prime}}(r),
\end{aligned}
$$



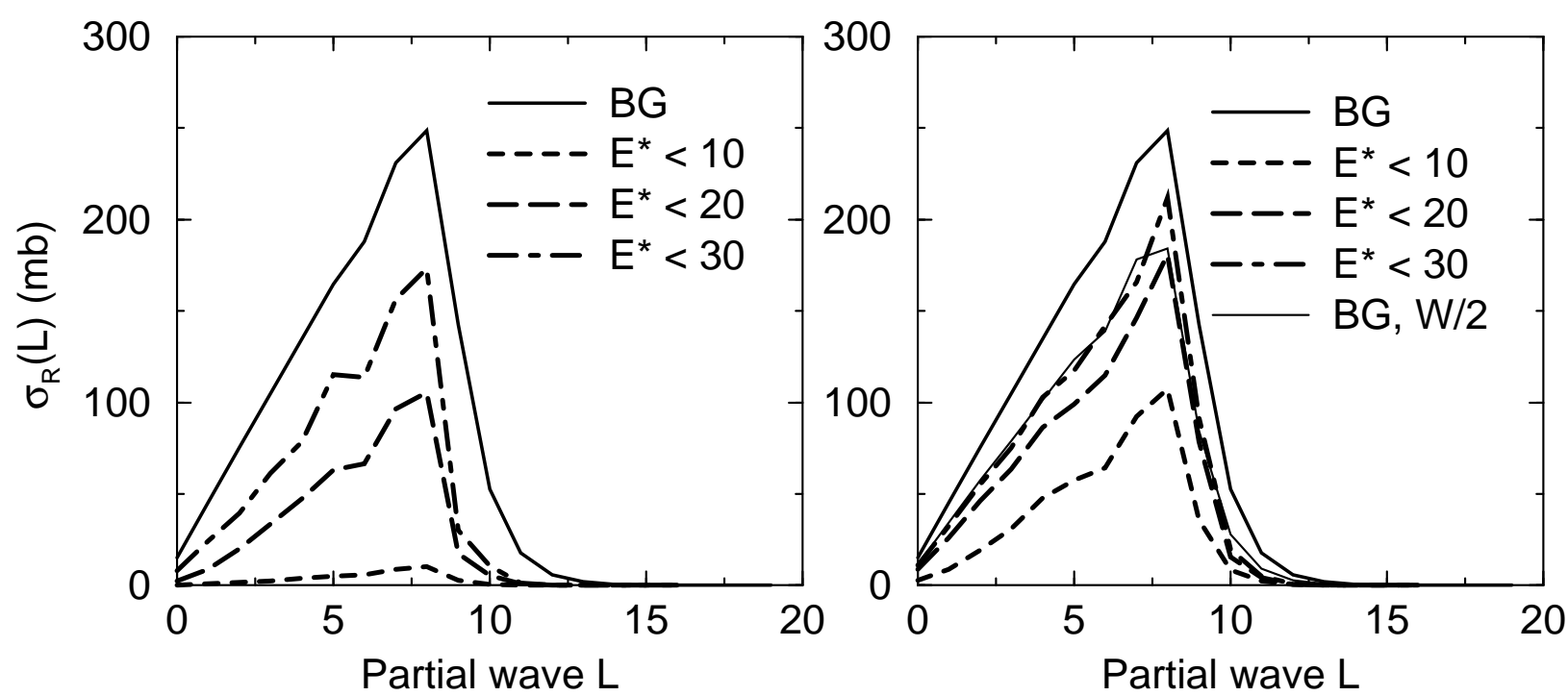

FIGURE 3. Single-particle (left) and RPA (right) partial wave reaction cross sections for neutrons incident on ${ }^{90} \mathrm{Zr}$ at $40 \mathrm{MeV}$, for various upper limits $E^{*}$ for excited states in $\mathrm{MeV}$.

where

$$
\begin{aligned}
& F_{L S J}^{\mathscr{J}}\left(l_{c} s_{c} j_{c} I_{c} ; l_{c^{\prime}} S_{c^{\prime}} j_{c^{\prime}} I_{c^{\prime}}\right)=\frac{1}{\sqrt{4 \pi}}(-1)^{j_{c^{\prime}}+I_{c}+\mathscr{J}+l_{c}} \\
& \times \hat{l_{c}} \hat{l_{c^{\prime}}} \hat{j_{c}} \hat{j_{c^{\prime}}} \hat{L} \hat{J}\left\{\begin{array}{ccc}
j_{c} & j_{c^{\prime}} & J \\
I_{c^{\prime}} & I_{c} & \mathscr{J}
\end{array}\right\} \\
& \times\left(\begin{array}{ccc}
l_{c} & l_{c^{\prime}} & L \\
0 & 0 & 0
\end{array}\right)\left\{\begin{array}{lll}
l_{c} & l_{c^{\prime}} & L \\
s_{c} & s_{c^{\prime}} & S \\
j_{c} & j_{c^{\prime}} & J
\end{array}\right\}
\end{aligned}
$$

We also leave spin-dependent forces for later work, so we use $\left(s=1 / 2\left\|\mathscr{S}_{0}\right\| s=1 / 2\right)=\sqrt{2}$.

We perform coupled inelastic channels calculations using FRESCO [6] for incident neutrons on ${ }^{90} \mathrm{Zr}$ at at $E_{\text {lab }}(n)=40 \mathrm{MeV}$. We use different upper limits on the RPA excitation energies, $E^{*}<10,20$ and $30 \mathrm{MeV}$, and compare the RPA results with those using uncorrelated particle-hole states for their spectra. The largest coupled-channels calculation included a maximum of 1277 partial waves. The inelastic cross sections obtained are shown in Fig. 2. We see now a considerable reduction in the remaining real dipole modes, how the $3^{-}$collective strength moves very much lower in energy, and how in the other channels there are strong giant resonances between 10 and $20 \mathrm{MeV}$.

From the coupled channels calculations, we extract the elastic S-matrix elements $S_{l}$. From these, we can calculate the elastic scattering angular distributions $\sigma_{\mathrm{el}}(\theta)$, and also the partial wave reaction cross sections

$$
\sigma_{R}(l)=\frac{\pi}{k^{2}}(2 l+1)\left[1-\left|S_{l}\right|^{2}\right] .
$$

We remark that we could use these partial reaction cross sections $\sigma_{R}(l)$ directly in Hauser-Feshbach models if they could be parameterised for their dependence on incident energy $E$ and partial wave $l$. This is because, the theory of non-overlapping resonances gives

$$
1-\left|S_{l}\right|^{2}=2 \pi\langle\Gamma\rangle / D
$$

(14) where $\langle\Gamma\rangle$ is the average widths of compound nucleus resonances, and $D$ is their mean level spacing. Thus Hauser-Feshbach models, with an independent specification of level densities, can use $\sigma_{R}(l)$ to determine their needed mean $\mathrm{CN}$ widths $\langle\Gamma\rangle$.

The conventional method is to fit $\sigma_{\mathrm{el}}(\theta)$ and find an elastic optical potential that reproduces this angular distribution to sufficient accuracy, and therefore has optical S-matrix elements $S_{l}$ that can be used in Eq. (16) to give the required average widths.

Our results for the partial wave reaction cross sections are shown in Fig. 3, with single-particle excitation model results on the left, and results from the RPA models on the right. In both cases, the dashed, long dashed and dotdashed curves show the increasing reaction cross sections from excitation of states up to 10,20 and $30 \mathrm{MeV}$, respectively, By comparing the left and right figures, we see that the correlations that are included only in the RPA model tend to increase the total reaction cross sections, and that this occurs over all the excitation energy ranges.

Also shown on the right of Fig. 3 by the thick solid line is the full reaction cross section from the fitted global neutron potential of Becchetti and Greenless [7], and by the thin solid line the results from that potential with its imaginary components reduced by $50 \%$. We see that 
even with $E^{*}=30 \mathrm{MeV}$ excited states in the model we fall short of the empirical reaction cross section, and in fact achieve results equivalent to only $50 \%$ of the empirical imaginary potential.

\section{DISCUSSION}

The fraction of the imaginary part of the optical potential that can be explained by the coupling to the excited states up to $30 \mathrm{MeV}$ in the RPA model, at $50 \%$, is comparable to results obtained previously from nuclear matter approaches [8]. There are several reasons for this only partial success.

First, this RPA structure model may still be improved in accuracy. The first $2^{+}$state in ${ }^{90} \mathrm{Zr}$, for example, is at $2.2 \mathrm{MeV}$ not at $4.6 \mathrm{MeV}$ as given by the RPA model. A systematic comparison of RPA predictions for $2^{+}$energies has recently been published [9].

Second, we have only included couplings to and from the ground state, not couplings between pairs of excited states. It is our experience [10] with halo breakup reactions, for which the basic excitation mechanisms are similar to the particle-hole processes described here, that the couplings between excited states (what are there called 'continuum-continuum' couplings) are very important and can change excitation cross sections by more than a factor of two.

Third, our RPA model gives a level density far short of the actual level density of compound nucleus states: it only models the 'particle-hole doorway states' that can be reached from the ground state by one application of the particle-hole operators $\mathscr{A}_{I M}^{\dagger}(p, \tilde{h})$. The doorway states can themselves be coupled to two-particle-twohole states, and flux removed by these $2 \mathrm{p} 2 \mathrm{~h}$ states will be a further contribution to the elastic optical potential. These $2 \mathrm{p} 2 \mathrm{~h}$ states should ideally be included also in the structure calculations, in what would then be called the 'Second RPA' framework [11].

Fourth, we have not included any transfer reactions, such as proton transfers leading to virtual or real production of deuterons. These are known [12] to contribute to nucleon optical potentials.

Since this calculation uses the structure results of the UNEDF collaboration [1], we expect the accuracy of the excited state energies and transition strengths to improve in the future. We will soon be including couplings between RPA excited states in our coupled-channels calculations, but including $2 \mathrm{p} 2 \mathrm{~h}$ states and transfer reactions is a project for the longer term. In the meantime, we argue that the $1 \mathrm{p} 1 \mathrm{~h}$ doorway states have similar ratios $\langle\Gamma\rangle / D$ as the $2 \mathrm{p} 2 \mathrm{~h}$ states, at least concerning their escape widths, because the later states fractionate the doorway state strength in proportion to their increased number of levels. Since in the Hauser-Feshbach calculations the imaginary strength is proportional to just to this $\langle\Gamma\rangle / D$ ratio, we do not expect very large effects of the $2 \mathrm{p} 2 \mathrm{~h}$ couplings on the imaginary part of the optical potential. Eventually we hope to test this expectation by means of the much larger and more detailed calculations that are now possible with modern parallel computers.

\section{ACKNOWLEDGMENTS}

This work was performed under the auspices of the U.S. Department of Energy by Lawrence Livermore National Laboratory under Contract DE-AC52-07NA27344, and under SciDAC Contract DE-FC02-07ER41457.

\section{REFERENCES}

1. G.F. Bertsch, J. Phys. Conf. Series 78 (2007) 012005; G. Bertsch et al., Building a Universal Nuclear Energy Density Functional, unedf . org.

2. M. Dupuis, University of Bordeaux Thesis (2006).

3. J. Decharge and D. Gogny, Phys. Rev. C 21, 1568 (1980).

4. G. Love, in The $(p, n)$ Reaction and the Nucleon-Nucleon Force, C. D. Goodman et al., Plenum, 1980.

5. G. Bertsch, J. Borysowicz, H. McManus, and W.G. Love, Nucl. Phys. A 284 (1977) 399.

6. I.J. Thompson, Comp. Phys. Rep., 7 (1988) 167.

7. F.D. Becchetti and G.W. Greenlees, Phys. Rev. 182 (1969) 1190.

8. F. Osterfeld, J. Wambach and V.A. Madsen, Phys. Rev. 23 (1981) 179.

9. G.F. Bertsch, M. Girod, S. Hilaire, J.-P. Delaroche, H. Goutte and S. Péru, Phys. Rev. Lett. 99, 032502 (2007).

10. F.M. Nunes and I.J. Thompson, Phys. Rev. C 59 (1999) 2652.

11. P. Papakonstantinou and R. Roth, arXiv:0709.3167v1.

12. R.S. Mackintosh, A.A. Ioannides and I.J. Thompson, Phys. Letts. 178B (1986) 1. 